\title{
Locally uniformly rotund renormings of the spaces of continuous functions on Fedorchuk compacts
}

\author{
S.P.Gul'ko ${ }^{a}$, A.V.Ivanov ${ }^{b}$, M.S.Shulikina $^{c}$, S.Troyanskiø \\ ${ }^{a}$ Faculty of Mechanics and Mathematics, Tomsk State University, Prospect \\ Lenina 36634010 Tomsk, Russian Federation E-mail address: gulko@math.tsu.ru \\ ${ }^{b}$ Institute of Applied Mathematical Research of Karelian Research Centre, Rus- \\ sian Academy of Sciences, Russian Federation, \\ E-mail address: alvlivanov@krc.karelia.ru \\ ${ }^{c}$ Department of Mathematics and Computer Science, Tomsk Polytechnic Uni- \\ versity, Russian Federation, E-mail address:shulikinams@tpu.ru \\ ${ }^{d}$ Institute of Mathematics and Informatics, Bulgarian Academy of Science, bl.8, \\ acad. G. Bonchev str., 1113 Sofia, Bulgaria and Departamento de Matemáticas, \\ Universidad de Murcia, Campus de Espinardo, 30100 Murcia, Spain, E-mail ad- \\ dress:stroya@um.es
}

\begin{abstract}
We show that $C(X)$ admits an equivalent pointwise lower semicontinuous locally uniformly rotund norm provided $X$ is Fedorchuk compact of spectral height 3 . In other words $X$ admits a fully closed map $f$ onto a metric compact $Y$ such that $f^{-1}(y)$ is metrizable for all $y \in Y$. A continuous map of compacts $f: X \rightarrow Y$ is said to be fully closed if for any disjoint closed subsets $A, B \subset X$ the intersection $f(A) \cap f(B)$ is finite. For instance the projection of the lexicographic square onto the first factor is fully closed and all its fibers are homeomorphic to the closed interval.
\end{abstract}

\section{INTRODUCTION}

Let us recall that a Banach space $E$ (or the norm in $E$ ) is said to be locally uniformly rotund (LUR for short) if $\lim _{n}\left\|x_{n}-x\right\|=0$ whenever

$$
\lim _{n}\left\|\left(x_{n}+x\right) / 2\right\|=\lim _{n}\left\|x_{n}\right\|=\|x\| .
$$

The spaces with this property are at the core of renorming theory in Banach spaces and consequently have been extensively studied (see, for example, [3] and [14] and its reference). It is well known that the spaces with a LUR norm have the Kadec property, that is on the unit sphere of $E$ the norm topology and the weak topology coincide. The LUR renorming techniques for a Banach space developed until now, are based in two different approaches. In the first one, for enough convex functions on the Banach space are constructed to apply Deville's master lemma (see the decomposition method [3, Chapter 7, Lemma 1.1p.279]), sometimes adding an iteration processes and Banach's contraction mapping theorem, to finally get an

\footnotetext{
${ }^{1}$ First three authors was supported by the Russian Foundation for Basic Research in the framework of the scientific project N 17-51-18051. The second author was also supported by state order to the Karelian Research Centre of the Russian Academy of Sciences (Institute of Applied Mathematical Research KarRC RAS). The forth author was partially supported by MTM 201786182-P (AEI/FEDER,UE) and by Bulgarian National Scientific Fund under Grant DFNI/Russia, 01/06/23.06.2017.
} 
equivalent LUR norm. Originally this method use the powerful method of projectional resolutions of the identity(PRI for short) The second one is based to a characterization of those Banach spaces that admit a LUR renorming by means of a linear topological condition of covering type [13]. The existence of such norm is deduced from the existence of some maps acting from normed space $E$ into metric space $Y$. This maps admit some covering properties (see [14]). We present a particular result in this direction which we use latter. This is nonlinear (convex) version for LUR renorming of Banach spaces with strong Markushevich basis.

Theorem 1.1. Assume that there is a locally bounded map $\Phi$ from a normed space $E$ into $c_{0}(\Gamma)$ for some set $\Gamma$ such that:

(i) for every finite set $A \subset \Gamma$ is specified a separable subspace $Z_{A}$ of $E$ such that:

(a) $Z_{A} \subset Z_{B}$ whenever $A \subset B \subset \Gamma$;

(b) $x \in \overline{\operatorname{span} \cup_{n \in N} Z_{K_{n}}}\|\cdot\|$ whenever $x \in E$ and $\left\{K_{n}: n \in N\right\}$ is an increasing sequence (i.e. $K_{n} \subset K_{m}$ for $n<m$ ) of finite subsets of $\Gamma$ with $\cup_{n \in N} K_{n} \supseteq$ $\operatorname{supp}(\Phi x)=\{\gamma \in \Gamma: \Phi x(\gamma) \neq 0\}$

(ii) there exists norming subspace $F$ of $E^{*}$ such that for every $\gamma \in \Gamma$ the real function $\delta_{\gamma} \circ \Phi$ on $E$ is non-negative, convex and $\sigma(E, F)$-lower semi-continuous, where $\delta_{\gamma}$ is the Dirac measure on $\Gamma$ at $\gamma$.

Then $E$ admits an equivalent $\sigma(E, F)$-lower semi-continuous LUR norm.

Remark. Condition(b) is equivalent to

(b') for every $x \in E$ there exists an increasing sequence $\left\{K_{n}: n \in N\right\}$ of finite subsets of the support $\operatorname{supp}(\Phi x)$ with $x \in \overline{\operatorname{span} \cup_{n \in N} Z_{K_{n}}}\|\cdot\|$.

Proof. Let us consider the condition

(iii) for every $x \in E$ there exists separable subspace $Z(x)$ of $E$ with

$$
x \in{\overline{\operatorname{span} \cup\left\{Z\left(x_{n}\right): n \in N\right\}}}^{\|\cdot\|}
$$

whenever $\left\{x_{n}: n \in N\right\}$ is a sequence in $E$ with $\lim _{n}\left\|\Phi x_{n}-\Phi x\right\|_{\infty}=0$.

In [14, Theorem 1.15, Theorem 2.14, Corollary 1.21, Theorem 3.28, Corollary 4.34 ] is shown that (ii) and (iii) imply existence in $E$ an equivalent $\sigma(E, F)$-lower semi-continuous LUR norm. So in order to prove the former Theorem we have to show $($ i) $=>$ (iii).

Let us mention that from (a) it follows that

$$
\operatorname{span} \cup_{n \in N} Z_{A_{n}}=\operatorname{span} \cup_{n \in N} Z_{B_{n}}
$$

for any increasing sequences $\left\{A_{n}: n \in N\right\},\left\{B_{n}: n \in N\right\}$ of finite subsets of $\Gamma$ with $\cup_{n \in N} A_{n}=\cup_{n \in N} B_{n}$. So we can define $Z_{A}$ for any countable subset $A$ of $\Gamma$ by setting

$$
Z_{A}=\overline{\operatorname{span} \cup_{n \in N} Z_{A_{n}}}\|\cdot\|
$$

if $A=\cup_{n \in N} A_{n}$ where $\left\{A_{n}: n \in N\right\}$ an increasing sequence of finite subsets of $\Gamma$. Clearly (a) holds for countable sets as well.

Set $Z(x)=Z_{\operatorname{supp}(\Phi x)}$. Let $\left\{x_{n}: n \in N\right\}$ be a sequence in $E$ with

$$
\lim _{n}\left\|\Phi x_{n}-\Phi x\right\|_{\infty}=0
$$

for some $x \in E$. Using (b') we can find an increasing sequence $\left\{K_{m}: m \in N\right\}$ of finite subsets of $\operatorname{supp}(\Phi x)$ such that

$$
x \in \overline{\operatorname{span} \cup_{m \in N} Z_{K_{m}}}\|\cdot\|
$$


Set $B_{n}=\operatorname{supp}\left(\Phi x_{n}\right)$. Fix $m \in N$. From (1.2) it follows that $K_{m} \subset B_{n}$ for all large enough $n$. Hence $Z_{K_{m}} \subset Z_{B_{n}}=Z\left(x_{n}\right)$ for all large enough $n$. So

$$
\cup_{m \in N} Z_{K_{m}} \subset \cup_{n \in N} Z\left(x_{n}\right) .
$$

This and (1.3) imply(1.1).

Example 1.2. Assume that $E$ has a srtrong Markushevich basis $\left\{e_{\gamma}: \gamma \in \Gamma\right\}$ with conjugate system $\left\{e_{\gamma}^{*}: \gamma \in \Gamma\right\}$, that is $e_{\beta}^{*}\left(e_{\gamma}\right)=\delta_{\beta \gamma}$, and for every $x \in E$ we have

$$
x \in{\overline{\operatorname{span}\left\{e_{\gamma}: e_{\gamma}^{*}(x) \neq 0, \gamma \in \Gamma\right\}}}^{\|\cdot\|} .
$$

For finite set $A \subset \Gamma$ set $Z_{A}=\operatorname{span}\left\{e_{\gamma}: \gamma \in A\right\}$ and define $\Phi: E \rightarrow c_{0}(\Gamma)$ by formula $\Phi x(\gamma)=\left|e_{\gamma}^{*}(x)\right|$. From the definition of strong Markushevich basis for every $x \in E$ we have

$$
x \in \overline{\operatorname{span}\left\{e_{\gamma}: \Phi x(\gamma)=\left|e_{\gamma}^{*}(x)\right| \neq 0, \gamma \in \Gamma\right\}^{|\cdot|} .} .
$$

So $\Phi$ satisfies the hypothesis of the former Theorem.

When Banach space $E$ admits PRI in the similar way we can construct map $\Phi$.

Lot of papers are devoted to find different classes of Hausdorff compact spaces (compacts) $X$ for which $C(X)$ admits an equivalent pointwise lower semi-continuous LUR norm. Moving on now to topological properties, we say that a compact space $X$ is Eberlein if it is homeomorphic to a weakly compact subset of a Banach space. Equivalently, $X$ is Eberlein if and only if $C(X)$ is a weakly compactly generated space. The space $X$ is called Talagrand or Gul'ko compact if $C(X)$ is weakly $K$ analytic or weakly countably determined, respectively. For a full treatment of these concepts, we refer the reader to e.g., [3]. It turns out that if $X$ hails from one of these three classes of compact spaces then it can be treated as a subset of a pointwise compact cube $[0,1]^{\Gamma}$, in such a way that, given $t \in X$, its coordinates $t(\gamma), \gamma \in \Gamma$ behave according to certain rules. For instance, $X$ is Eberlein if and only if we can find such an embedding with the property that for any $t \in X$ and $\varepsilon>0$, there are only finitely many $\gamma \in \Gamma$ satisfying $|t(\gamma)| \geq \varepsilon$. Similarly, spaces from the three corresponding classes of Banach spaces may be endowed with 'coordinate systems', which permit the spaces to be carefully analyzed in ways which are not feasible in a fully general, non-separable setting. Every compact space $X$ from the classes above shares the property that $X$ may be embedded in $[0,1]^{\Gamma}$, such that given any $t \in X$, the support of $t$ is countable. In general, a space satisfying this property is called Corson compact. The space $X$ is called Valdivia compact if it is as above, but, in this case, only a pointwise dense subset of points of $X \subset[0,1]^{\Gamma}$ are required to have countable support. These classes have long been relevant to renorming theory. For example, it can be shown that if $X$ is Valdivia compact then $C(X)$ admits a pointwise lower semi-continuous LUR norm (cf. [3, Corollary VII.1.10]) . In all this cases $C(X)$ admits PRI. Of course all this result can be obtained directly from Theorem 1.1. We mention that in $C(X)$ spaces we have a canonical map to $c_{0}(\Gamma)$ (see e.g. [14]). Indeed if $X \subset[0,1]^{\Gamma}$ the uniform continuity of every $h \in C(X)$ allows us to define the oscillation map $\Omega: C(X) \rightarrow c_{0}(\Gamma)$ by formula

$$
\Omega(h)(\gamma)=\sup \left\{h(t)-h(s): t, s \in X,\left.(t-s)\right|_{\Gamma \backslash\{\gamma\}}=0\right\},
$$

where $h \in C(X)$. Map $\Omega$ was introduced(see [4]) looking for countable sets of coordinates which control a continuous function to obtain extensions of the theorem of Mibu. It is easy to see that $\Omega$ is a bounded map satisfying condition (ii) of Theorem 1.1. In $[14,2.7]$ is shown that $\Omega$ satisfies condition (iii) of Theorem 1.1 
when $X$ is Helly compact of monotone functions on $[0,1]$. In this way is proved that $C(X)$ is LUR renormable when $X$ is Helly compact. A generalization of this result when $X$ is a particular case of Rosenthal compacts can be found in[10], see also [12].

The aim of this note is to find a new class of compact spaces for which the corresponding space of continuous functions is LUR renormable.

The following definition goes back to V.V.Fedorchuk (see e.g. [5, II.1.6])

Definition 1.3. Continuous map of compacts $f: X \rightarrow Y$ is said to be fully closed if for every disjoint closed subsets $A$ and $B$ of $X$ the set $f(A) \cap f(B)$ is finite.

Our main result is next

Theorem 1.4. Let $X$ be a compact space admitting a fully closed map $f$ onto a metrizable compact $Y$ such that the fibers $f^{-1}(y)$ are metrizable for every $y \in Y$. Then $C(X)$ admits an equivalent pointwise lower semi-continuous LUR norm.

The above class of compact spaces is particular case of Fedorchuk compacts. The section 2 is devoted to this class. Now we give an example. Denote with $L$ the lexicographic square (the projection of this square onto the first factor is fully closed and all its fibers are homeomorphic to the closed interval). In [1] is shown that $C(L)$ is LUR renormable(for general case of totally ordered compacts see [9] and $[14])$.

Let $f: X \rightarrow Y$ be a continuous map of compacts. Given $y \in Y$ define

$$
o s c_{f^{-1}(y)}(h)=\sup _{s, t \in f^{-1}(y)}\{h(t)-h(s)\}=\operatorname{diam}\left(h\left(f^{-1}(y)\right)\right.
$$

for $h \in C(X)$. Clearly $\operatorname{osc}_{f}-1(y)(\cdot)$ is a pointwise lower semi-continuous semi-norm in $C(X)$ and $\operatorname{osc}_{f-1}(y)(\cdot) \leq 2\|\cdot\|_{\infty}$.

We introduce fiberwise oscillation map $\Omega_{f}: C(X) \rightarrow l_{\infty}(Y)$ by formula

$$
\Omega_{f}(h)(y)=\operatorname{osc}_{f^{-1}(y)}(h)
$$

where $h \in C(X)$.

Since $\left\|\Omega_{f}(h)\right\|_{\infty} \leq 2\|h\|_{\infty}$ for all $h \in C(X)$ the map $\Omega_{f}$ is bounded. Since $\operatorname{osc}_{f^{-1}(y)}(\cdot)$ is a pointwise lower semi-continuous semi-norm in $C(X)$ and $\delta_{y} \circ$ $\Omega_{f}(h)=o s c_{f^{-1}(y)}(h)$ we get that $\Omega_{f}$ satisfies condition (ii) of Theorem 1.1. In sections 2 and 3 we show that if $f$ a fully closed map satisfying the conditions of Theorem 1.4 then $\Omega_{f}$ maps $C(X)$ into $c_{0}(Y)$ and satisfies condition (i) of Theorem 1.1.

\section{FEDORCHUK COMPACTS}

The class of Fedorchuk compact spaces was defined in 1984 [11] with the purpose of clarifying the limits of the application of the method of resolutions, which was developed by Fedorchuk (see [5]) and showed exceptional efficiency in constructing counterexamples in general topology. The definition of this class is inextricably linked with the concept of a fully closed mapping introduced by Fedorchuk in the process of developing the method mentioned above. The original definition of fully closed mapping was cumbersome. Later, his author obtained a number of unobvious equivalent formulations (see [5, II. 1.6]). The shortest of them for mappings of Hausdorff compact spaces is the Definition 1.3 above.

Definition 2.1. Hausdorff compact space $X$ is called a Fedorchuk compact (or an $F$-compact) if there exists a well-ordered continuous inverse system (an F-system) 
$S=\left\{X_{\alpha}, \pi_{\beta}^{\alpha}: \alpha, \beta \in \gamma\right\}$ (here $\gamma$ is an ordinal number) giving in the limit $X$, in which $X_{0}$ is the point, all the neighboring projections $\pi_{\alpha}^{\alpha+1}$ are fully closed $(\alpha+1<$ $\gamma)$, and the inverse images of the points $\left(\pi_{\alpha}^{\alpha+1}\right)^{-1}(x)$ are metrizable for any $x \in X_{\alpha}$. The spectral height sh $(X)$ of an $F$-compact $X$ is the smallest possible length $\gamma$ of such a system.

We consider only the Fedorchuk compacts of spectral height 3 . If $X$ is an $F$ compact and $\operatorname{sh}(X)=3$, then $X$ is the limit of an $F$-system consisting of three spaces: $X_{0}, X_{1}$ and $X_{2}$. The limit of this system coincides with $X_{2}$, from which it follows that the compact space $X=X_{2}$ is non-metrizable, since otherwise it could be obtained as the limit of the $F$-system of two compacts: $X_{0}$ and $X_{2}$ (the map to a point is always fully closed). Consequently, for any Fedorchuk compact of spectral height 3 there exists a fully closed map $\pi_{1}^{2}: X=X_{2} \rightarrow X_{1}$ onto a metric compact space for which the sets $\left(\pi_{1}^{2}\right)^{-1}(x), x \in X_{1}$ are metrizable.

Thus, $F$-compacts of spectral height 3 can be characterized as non-metrizable compacts that admit a fully closed map onto a metric compact with metrizable fibers. Note that if such a fully closed map for a compact $X$ exists, then it is almost unique in the following sense: almost all (that is, all but perhaps a countable set) nontrivial fibers of any two such maps coincide (see [6]). Remark, that only a point is an $F$-compact of spectral height 1 and $F$-compacts of spectral height 2 are all non-trivial metric compact spaces.

As noted in the introduction, the lexicographic square $L$ is an $F$-compact of spectral height 3. The classical space "two arrows" (the lexicographic product of unit segment and two-point set $[0,1] \times\{0,1\})$ also is $F$-compact of spectral height 3 (the standard projection of this space onto the segment is a fully closed map) as well as the space "Alexandroff double circle" (the projection of "double circle" onto the circle is fully closed).

To the class of $F$-compacts of spectral height 3 belongs a series of various counterexamples constructed by Fedorchuk. Here, first of all, we should mention a group of compacts with non-coinciding dimensions. Among them is the famous example of a two-dimensional compact that has no partitions of lower dimension (see [2], page 314), and also a homogeneous separable compact $X$ with the first axiom of countability with $\operatorname{dim}(X)=1<\operatorname{ind}(X)=2$ and a perfectly normal compact (constructed under the assumption of $C H$ ) with same values of the dimensions $\mathrm{dim}$ and ind (see [5, III. 3.6 and 3.10]).

We will need the following characteristic property of fully closed map obtained by Fedorchuk [5]. Let $f: X \rightarrow Y$ be a continuous map, and $A$ be an arbitrary subset of $Y$. Consider a partition of a compact $X$ whose nontrivial elements are sets $f^{-1}(y)$ for $y \in Y \backslash A$. Let $Y_{A}$ be the quotient space corresponding to this partition (with respect to $f$ ), that is

$$
Y_{A}=\left\{f^{-1}(y): y \in Y \backslash A\right\} \cup\{\{x\}: f(x) \in A\} .
$$

Let $f_{A}: X \rightarrow Y_{A}$ be a quotient map, and let $\pi_{A}: Y_{A} \rightarrow Y$ be the unique map for which $f=\pi_{A} \circ f_{A}$.

Proposition 2.2. [5, II. 1.6]. The map $f$ is fully closed if and only if for each $A \subset Y$ the space $Y_{A}$ is Hausdorff.

In $[5, \mathrm{II}, 1.7,1.10]$ it is also shown that for a fully closed map $f$ the maps $f_{A}$ and $\pi_{A}$ are also fully closed for any $A \subset Y$.

Proposition 2.3. [5, II. 3.10]. Let $f: X \rightarrow Y$ be a fully closed map of the compact $X$ onto a metric compact $Y$ with metrizable fibers $f^{-1}(y), y \in Y$. The 
compact $X$ is metrizable if and only if the set of nontrivial fibers (i.e. the fibers $f^{-1}(y)$ that is not a singleton) of $f$ is countable.

\section{PROOF OF THEOREM 1.4.}

The following assertion gives a characterization of fully closed maps, which plays an important role in what follows.

Proposition 3.1. The map $f: X \rightarrow Y$ of compacts is fully closed if and only if for any continuous map $g: X \rightarrow K$ into a metric compact space $K$ and any $\varepsilon>0$ the set $H_{g, \varepsilon}=\left\{y \in Y: \operatorname{diam}\left(g\left(f^{-1}(y)\right)\right) \geq \varepsilon\right\}$ is finite.

Proof. Necessity. Let $f$ be fully closed. This means that for any disjoint closed subsets $A, B \subset X$ the intersection $f(A) \cap f(B)$ is finite. Suppose that there exists a continuous map $g: X \rightarrow K$ to the metric compact $K$ and $\varepsilon>0$ such that $H_{g, \varepsilon}$ is infinite. Consider the infinite family of closed subsets of $K$ indexed by $y$ :

$$
G=\left\{g\left(f^{-1}(y)\right): y \in H_{g, \varepsilon}\right\} .
$$

Let $F$ be an accumulation point of the family $G$ in the space $\exp (K)$ of nonempty closed subsets of $K$ endowed with the Hausdorff metric. It is obvious that $\operatorname{diam}(F) \geq$ $\varepsilon$. We take in $F$ two distinct points $x_{1}, x_{2}$ and their neighborhoods $O_{x_{1}}, O_{x_{2}}$ with disjoint closures. By the choice of $F$, there exists an infinite subset $D \subset H_{g, \varepsilon}$ such that $g\left(f^{-1}(y)\right) \cap O_{x_{i}} \neq \emptyset$ for any $y \in D$ for $i=1,2$. Consider disjoint closed subsets $A_{i}=g^{-1} \overline{O_{x_{i}}}, i=1,2$ in the space $X$. We have $D \subset f\left(A_{1}\right) \cap f\left(A_{2}\right)$, which contradicts that $f$ is fully closed.

Sufficiency. Let $f$ be not fully closed, that is, there exist disjoint closed subsets $A, B$ in $X$ such that the intersection $E=f(A) \cap f(B)$ is infinite. Let $g: X \rightarrow[0,1]$ be a continuous function on $X$ that separates $A$ and $B$. Then the set $H_{g, 1}$ contains $E$ and, consequently, is infinite.

Corollary 3.2. For any fully closed map of compacts $f: X \rightarrow Y$ the fiberwise oscillation map $\Omega_{f}$ maps $C(X)$ into $c_{0}(Y)$.

Let $f: X \rightarrow Y$ be a fully closed map of compacts. Given $A \subset Y$ define

$$
Z_{A}=\left\{h \in C(X): \operatorname{supp}\left(\Omega_{f}(h)\right) \subset A\right\} .
$$

From the definition of $\Omega_{f}$ it follows that $Z_{A}$ is a closed subspace of $C(X)$.

Lemma 3.3. The spaces $Z_{A}$ and $C\left(Y_{A}\right)$ are isomorphically isometric. The linear operator $T_{A}: Z_{A} \rightarrow C\left(Y_{A}\right)$ defined by formula

$$
T_{A} h=h \circ f_{A}^{-1}
$$

give the isometry, i.e.

$$
\left\|T_{A} h\right\|_{\infty}=\|h\|_{\infty} .
$$

Moreover for every $y \in A$ and $h \in Z_{A}$ we have

$$
T_{A} h\left(\pi_{A}^{-1}(y)\right)=h\left(f^{-1}(y)\right) .
$$

Proof. We have

$$
Y_{A}=\left\{f^{-1}(y): y \in Y \backslash A\right\} \cup\{\{x\}: f(x) \in A\} .
$$

Pick $h \in Z_{A}$. Then $\operatorname{osc}_{f^{-1}(y)}(h)=0$ if $y \in Y \backslash A$. So $\left(T_{A} h\right)\left(f^{-1}(y)\right)=h \circ$ $f_{A}^{-1}\left(f^{-1}(y)\right)=h(x)$ (here $f^{-1}(y)$ is a point of $Y_{A}$ ) for every $x \in f^{-1}(y)$ and $y \in Y \backslash A$. If $f(x) \in A$ then $\left(T_{A} h\right)(\{x\})=h\left(f_{A}^{-1}(\{x\})\right)=h(x)$. 
This implies that $T_{A} h$ is a real single valued and continuous function on $Y_{A}$. Moreover (3.3) holds.

Since $f=\pi_{A} \circ f_{A}$ we get $f^{-1}(y)=f_{A}^{-1}\left(\pi_{A}^{-1}(y)\right)$. So for $h \in Z_{A}$ and $y \in A$

$$
h\left(f^{-1}(y)\right)=h\left(f_{A}^{-1}\left(\pi_{A}^{-1}(y)\right)\right)=T_{A} h\left(\pi_{A}^{-1}(y)\right) .
$$

The main result of this section is

Proposition 3.4. Let $f: X \rightarrow Y$ be a fully closed map of compact $X$ onto a metric compact $Y$ with metrizable fibers $f^{-1}(y), y \in Y$. Let $K$ be a finite subset of a countable set $A \subset Y$. Then for every $h \in Z_{A}$ we have

$$
\operatorname{dist}\left(h, Z_{K}\right)=\inf \left\{\|h-g\|_{\infty}: g \in Z_{K}\right\} \leq\left\|\left.\Omega_{f}(h)\right|_{A \backslash K}\right\|_{\infty} .
$$

The proof is based on the properties of Fedorchuk compacts. For this reason at first, before the proof, we introduce some concepts and notations. In the subsequent arguments we need the concept of a small image of a set. Recall that a small image $f^{\sharp}(B)$ of the set $B \subset X$ under a map $f: X \rightarrow Y$ is the set $f^{\sharp}(B)=Y \backslash f(X \backslash B)$. Clearly $f^{\sharp}(B) \subset f(B)$. Let us mention that for any continuous map $f: X \rightarrow Y$ of compacts and any open subset $U \subset X$, a small image $f^{\sharp}(U)$ is open in Y, since the set $f(X \backslash U)$ is closed in $Y$ as a continuous image of a compact.

We denote with $\operatorname{Fr}(B)$ the topological boundary of the set $B$, that is $\operatorname{Fr}(B)=$ $\bar{B} \backslash \operatorname{int}(B)$.

Using the notations of section 2 we consider the fully closed map $\pi_{A}: Y_{A} \rightarrow Y$. Consider a partition of compact $Y_{A}$ whose nontrivial elements are the sets $\pi_{A}^{-1}(y)$ for $y \in Y \backslash K$. Let $Y_{A, K}$ be the quotient space corresponding to this partition, let $\pi_{A, K}: Y_{A} \rightarrow Y_{A, K}$ be the quotient map, and let $\varkappa_{A, K}: Y_{A, K} \rightarrow Y$ be the unique map for which $\pi_{A}=\varkappa_{A, K} \circ \pi_{A, K}$. By Proposition 2.3 we have that $Y_{A, K}$ is metrizable since the set of nontrivial fibers $\left\{\varkappa_{A, K}^{-1}(y): y \in K\right\}$ of the fully closed map $\varkappa_{A, K}$ is finite and all this fibers and $Y$ are metrizable. We fix a metric $d$ on $Y_{A, K}$ that is compatible with the topology. We denote with $B(u, \rho)=\left\{y \in Y_{A, K}\right.$ : $d(u, y)<\rho\}$ the open ball centered at $u$ with radius $\rho>0$.

Proof of Proposition 3.4. Pick $h \in Z_{A}$ and set

$$
s=\left.|| \Omega_{f}(h)\right|_{A \backslash K} \|_{\infty}, g=T_{A} h .
$$

So $g=h \circ f_{A}^{-1} \in C\left(Y_{A}\right)$. Set $D=A \backslash K$. Enumerate the points $D$ by the natural numbers: $D=\left\{y_{n}: n \in N\right\}$ and denote by $u_{n}$ the unique point in $Y_{A, K}$ with $\varkappa_{A, K}\left(u_{n}\right)=y_{n}$. Set $E=\left\{u_{n}: n \in N\right\}$.

Fix $c>1$. For every $n \in N$ choose an open neighborhood $O_{y_{n}}$ of $\pi_{A}^{-1}\left(y_{n}\right)$ in $Y_{A}$ in such a way that

$$
\operatorname{diam}\left(g\left(O_{y_{n}}\right)\right)<c \cdot \operatorname{diam}\left(g\left(\pi_{A}^{-1}\left(y_{n}\right)\right) .\right.
$$

From (3.4) we get $\operatorname{diam}\left(g\left(\pi_{A}^{-1}\left(y_{n}\right)\right)\right)=\operatorname{diam}\left(h\left(f^{-1}\left(y_{n}\right)\right)\right)$. Since $y_{n} \in A \backslash K$ we get $\operatorname{diam}\left(h\left(f^{-1}\left(y_{n}\right)\right)\right) \leq s$. So

$$
\operatorname{diam}\left(g\left(O_{y_{n}}\right)\right)<c s .
$$

Claim 1. There exists a sequence of open sets $\left\{U_{n}: n \in N\right\}$ in $Y_{A, K}$ such that $E \subset \cup_{n \in N} U_{n}$, the closures of $U_{n}$ are pairwise disjoint, $\overline{U_{n}} \subset \pi_{A, K}^{\sharp}\left(O_{y_{n}}\right), \operatorname{Fr}\left(U_{n}\right) \cap$ $E=\emptyset$ and $\operatorname{diam}\left(\overline{U_{n}}\right)<\min \{c s, 1 / n\}$. 
Proof of Claim 1. We construct the open sets $U_{n}, n \in N$ by recursion as follows.

Step 1. Clearly $u_{1} \in \pi_{A, K}^{\sharp}\left(O_{y_{1}}\right)$. Taking into account that $E$ is countable, we can choose an open neighborhood $U_{1}$ of the point $u_{1}$ such that $\overline{U_{1}} \subset \pi_{A, K}^{\sharp}\left(O_{y_{1}}\right), \operatorname{Fr}\left(U_{1}\right) \cap$ $E=\emptyset$ and $\operatorname{diam}\left(\overline{U_{1}}\right)<\min \{c s, 1\}$. As $U_{1}$ we can take an open ball $B\left(u_{1}, \rho\right)$ centered at $u_{1}$ with a sufficiently small radius $\rho \neq d\left(u_{1}, u_{k}\right), k \in N$. If $y \in \operatorname{Fr}\left(U_{1}\right)$ then $d\left(u_{1}, y\right)=\rho$. Therefore $\operatorname{Fr}\left(U_{1}\right) \cap E=\emptyset$.

Assume that the sets $U_{k}$ are already constructed for all $k<n$ such that their closures are disjoint, $\overline{U_{k}} \subset \pi_{A, K}^{\sharp}\left(O_{y_{k}}\right), \operatorname{Fr}\left(U_{k}\right) \cap E=\emptyset$, $\operatorname{diam}\left(\overline{U_{k}}\right)<\min \{c s, 1 / k\}$ and $u_{m} \in \cup_{k<n} U_{k}$ for $m<n$.

Step $n$. If $u_{n} \in \cup_{k<n} U_{k}$, then put $U_{n}=\emptyset$. Otherwise $u_{n} \notin \cup_{k<n} \overline{U_{k}}$ and we construct a neighborhood $U_{n}$ of the point $u_{n}$ as in step 1 such that $\overline{U_{n}} \subset$ $\pi_{A, K}^{\sharp}\left(O_{y_{n}}\right) \backslash \cup_{k<n} \overline{U_{k}}, \operatorname{Fr}\left(U_{n}\right) \cap E=\emptyset, \operatorname{diam} \overline{U_{n}}<\min \{c s, 1 / n\}$.

Set $G=Y_{A, K} \backslash \cup_{n \in N} U_{n}$ and $W=\pi_{A, K}^{-1}\left(\cup_{n \in N} U_{n}\right)$. The map $\pi_{A, K}$ is one-to-one on $Y_{A} \backslash W$ and $\pi_{A, K}\left(Y_{A} \backslash W\right)=G$. Define a real function on $G$ by formula

$$
r=g \circ\left(\left.\pi_{A, K}^{-1}\right|_{G}\right) \text {. }
$$

Set

We have

$$
a_{n}=\inf \left(g\left(O_{y_{n}}\right)\right), b_{n}=\sup \left(g\left(O_{y_{n}}\right)\right) .
$$

$$
b_{n}-a_{n}=\operatorname{diam}\left(g\left(O_{y_{n}}\right)\right)<c s .
$$

Thus $r\left(F r\left(U_{n}\right)\right) \subset\left[a_{n}, b_{n}\right]$ since $\overline{U_{n}} \subset \pi_{A, K}^{\sharp}\left(O_{y_{n}}\right)$.

Let us mention that from Proposition 3.1 and (3.6), (3.9) we get

$$
\lim _{n}\left(b_{n}-a_{n}\right)=0 .
$$

By the Tietze theorem on the extension of continuous functions defined on a closed subset of a metric space, there is a continuous function $r_{n}: \overline{U_{n}} \rightarrow\left[a_{n}, b_{n}\right]$, which is an extension of the function $\left.r\right|_{F r\left(U_{n}\right)}$.

Claim 2. The real function

$$
q(x)=\left\{\begin{array}{l}
r(x) \text { if } x \in G \\
r_{n}(x) \text { if } x \in U_{n}, n \in N
\end{array}\right.
$$

is continuous on $Y_{A, K}$.

Proof of Claim 2. Pick $x \in Y_{A, K}$. If $x \in \cup U_{n}$, then the continuity of $q$ at the point $x$ is obvious.

Let $x \in G \backslash \cup \overline{U_{n}}$. Let $z$ be the unique point of $Y_{A}$ for which $\pi_{A, K}(z)=x$. Fix $\varepsilon>0$. By the continuity of $\mathrm{g}$, there exists a neighborhood $V_{z}$ of $z$ such that for any $v \in V_{z}$ we have $|g(z)-g(v)|<\varepsilon$. It is clear that $x \in \pi_{A, K}^{\sharp}\left(V_{z}\right)$. Choose $k \in N$ such that $B(x, 1 / k) \subset \pi_{A, K}^{\sharp}\left(V_{z}\right)$. Using (3.10) we may assume in addition

$$
b_{n}-a_{n}<\varepsilon \text { for } n>k \text {. }
$$

We show that $|q(x)-q(w)|<2 \varepsilon$ for any $w \in B(x, 1 / 2 k) \backslash \cup_{n \leq 2 k} \overline{U_{n}}$ (the last set is an open neighborhood of the point $x$ ).

If $w \in G$, then there is a unique point $v \in V_{z}$ such that $\pi_{A, K}(v)=w$ and $q(w)=g(v)$. Consequently,

$$
|q(x)-q(w)|=|g(z)-g(v)|<\varepsilon .
$$


If $w \notin G$, then $w \in U_{n}$ for some $n>2 k$. Since $\operatorname{diam}\left(U_{n}\right)<1 / n \leq 1 / 2 k$ and $w \in B(x, 1 / 2 k)$, we get $U_{n} \subset B(x, 1 / k) \subset \pi_{A, K}^{\sharp}\left(V_{z}\right)$. By virtue of (3.11) we have

$$
\operatorname{diam}\left(g\left(O_{y_{n}}\right)\right)=b_{n}-a_{n}<\varepsilon .
$$

Let $t \in \pi_{A, K}^{-1}(w)$. Then $t \in O_{y_{n}}$, since by construction $U_{n} \subset \pi_{A, K}^{\sharp}\left(O_{y_{n}}\right)$. Therefore, $g(t) \in\left[a_{n}, b_{n}\right]$. By definition $q(w)=r_{n}(w) \in\left[a_{n}, b_{n}\right]$. Thus, $|g(t)-q(w)|<\varepsilon$. Since $t \in V_{z}$ we have

$$
|q(x)-q(w)|=|g(z)-q(w)| \leq|g(z)-g(t)|+|g(t)-q(w)|<2 \varepsilon .
$$

The continuity of $q$ at the point $x$ is proved.

It remains to consider the case when $x \in \operatorname{Fr}\left(U_{n}\right)$ for some $n$. In this case, we represent $Y_{A, K}$ as a union of two closed sets:

$$
Y_{A, K}=\overline{U_{n}} \cup\left(Y_{A, K} \backslash U_{n}\right) .
$$

The restriction of $q$ to $\overline{U_{n}}$ is continuous at the point $x$ by the definition of $q$. The continuity of the restriction of $q$ to $\left(Y_{A, K} \backslash U_{n}\right)$ at the point $x$ is proved by a verbatim repetition of the above arguments with $Y_{A, K}$ replaced by $\left(Y_{A, K} \backslash U_{n}\right), Y_{A}$ by $Y_{A} \backslash \pi_{A, K}^{-1}\left(U_{n}\right)$, and $U_{n}$ by the empty set. The continuity of $q$ at the point $x$ on $\overline{U_{n}}$ and $\left(Y_{A, K} \backslash U_{n}\right)$ implies continuity at the point $x$ on $Y_{A, K}$.

Set $p=q \circ \pi_{A, K} \circ f_{A}$. We have $p \in C(X)$.

Claim 3. We have

(a) $\|p-h\|_{\infty} \leq\left. c|| \Omega_{f}(h)\right|_{A \backslash K} \|_{\infty}$;

(b) supp $\Omega_{f}(p) \subset K \subset A$.

Proof of Claim 3. (a) Pick $x \in X$. Assume that and $\pi_{A, K}\left(f_{A}(x)\right) \in G$. From the definition of $q$ we get $p(x)=h(x)$.

Now let $\pi_{A, K}\left(f_{A}(x)\right) \notin G$. In this case $y=\pi_{A, K}\left(f_{A}(x)\right) \in U_{n}$ for some $n$. Since $\left.U_{n} \subset \pi_{A, K}^{\sharp}\left(O_{y_{n}}\right)\right)$ we get $f_{A}(x) \in O_{y_{n}}$ and $h(x)=g\left(f_{A}(x)\right) \in\left[a_{n}, b_{n}\right]$ by virtue of (3.9).

On the other hand, when $y \in U_{n}$

$$
p(x)=q(y)=r_{n}(y) \in\left[a_{n}, b_{n}\right],
$$

therefore $|p(x)-h(x)| \leq b_{n}-a_{n} \leq c s=\left.c|| \Omega_{f}(h)\right|_{A \backslash K} \|_{\infty}$. Thus $\|p-h\|_{\infty} \leq$ $\left.c|| \Omega_{f}(h)\right|_{A \backslash K} \|_{\infty}$.

(b) If $y \notin K \subset Y$, then $\varkappa_{A, K}^{-1}(y)=\{t\}$ is a singleton. Hence for any $x \in f^{-1}(y)$ $p(x)=q(t)$. Therefore $\operatorname{osc}_{f^{-1}(y)}(p)=0$ for $y \notin K$. Thus supp $\Omega_{f}(p) \subset K$.

Since $c$ is arbitrary number bigger than 1, (a) and (b) complete the proof of proposition.

In order to finish the proof of Theorem 1.4 it is enough to prove the following

Proposition 3.5. The map of the fiberwise oscillation $\Omega_{f}: C(X) \rightarrow c_{0}(Y)$ satisfies the conditions ( $a$ ) and (b) of (i) Theorem 1.1 for any fully closed map $f$ : $X \rightarrow Y$ of compact $X$ onto a metric compact $Y$ with metrizable fibers $f^{-1}(y), y \in$ $Y$.

Proof. Given a subset $A$ of $Y$ we define $Z_{A}$ as in (3.1). According Lemma3.3 $Z_{A}$ can be identify with $C\left(Y_{A}\right)$. If $A$ is finite or countable we get that $Y_{A}$ is metrizable by Proposition 2.3. Hence $C\left(Y_{A}\right)$ is separable. Evidently condition (a) holds. Pick $h \in C(X)$ and set

$$
K_{m}=\left\{y \in Y: \Omega_{f}(h)(y) \geq 1 / m\right\} .
$$


Applying Proposition 3.1 we get that $K_{m}, m \in N$ are finite. Clearly that $\left\{K_{m}\right.$ : $m \in N\}$ is an increasing sequence. From (3.5) we get $\operatorname{dist}\left(h, Z_{K_{m}}\right) \leq 1 / m$. So $\lim _{m} \operatorname{dist}\left(h, Z_{K_{m}}\right)=0$. From this it follows that

$$
h \in \overline{\operatorname{span} \cup_{m \in N} Z_{K_{m}}}\|\cdot\|_{\infty} .
$$

So (b') holds.

\section{References}

[1] G.Alexandrov, Spaces of continuous functions isomorphic to locally uniformly rotund Banach space, C. R. Acad. Bulg. Sci., 41, no 8 (1988), 9-12.

[2] P.S.Alexandrov, B.A.Pasynkov. Introduction into Dimension Theory. Moscow, "Nauka", 1973 [in Russian].

[3] R.Deville, G.Godefroy, V.,Zizler, Smoothness and renorming in Banach spaces, Pitman Monographs and Surveys in Pure and Appl. Math. 64, Longman Scientific and Technical, Longman House, Burnt Mill, Harlow, 1993.

[4] R.Engelking, On functions defined in Cartesian products, Fundamenta Math., 59 (1966), 221-231.

[5] V.V. Fedorchuk, Fully closed mappings and their applications, J. Math. Sci., 136(5) (2006), 4201- 4291.

[6] S.P.Gul'ko, A.V.Ivanov. On fully closed mappings of Fedorchuk compacta. Vestn. Tom. gos. un-ta. Matematika i mekhanika. 2017. No 50. P. 5-8.

[7] R.Haydon, Trees in renorming theory, Proc. London Math. Soc., 78 (1999), $541-584$.

[8] R.Haydon, Locally uniformly rotund norms in Banach spaces and their duals. J. Funct. Analysis, 254 (2008), 2023-2039.

[9] R.Haydon, J. Jayne, I. Namioka, C.A. Rogers, Continuous functions on totally ordered spaces that are compact in their order topologies. J. Funct. Analysis, 178 (2000), 23-63.

[10] R. Haydon, A.Moltó, J. Orihuela, Spaces of functions with countably many discontinuities, Israel J. Math., 158 (2007), 19-39.

[11] A.V. Ivanov, On Fedorchuk compacta, in: Mappings and Functors, Izd. Mosk. Univ.1984,pp. 31-40. (in Russian)

[12] J. F. Martínez, A.Moltó, J. Orihuela, S.Troyanski, On locally uniformly rotund renormings in C(K) spaces, Canadian J. Math., 62 (2010), 595 - 613.

[13] A.Moltó, J.Orihuela and S. Troyanski, Locally uniformly rotund renorming and fragmentability, Proc. London Math. Soc.,75 (1997), 619-640.

[14] A.Moltó, J. Orihuela, S.Troyanski, M. Valdivia, Non-linear transfer technique, Lect. Notes Math.,1951, Springer, Berlin, 2009. 